\title{
Urinary Neopterin in Patients with upper Gastrointestinal, Biliary and Pancreatic Carcinomas
}

\author{
Bohuslav Melichar ${ }^{12}$, Dagmar Solichová ${ }^{3}$, Iveta Svobodová ${ }^{3}$, Lubor Urbánek ${ }^{3}$, Karolina Melicharová $^{7}$ \\ Departments of 'Oncology \& Radiotherapy, 'Medicine, and 'Gerontology \& Metabolic Care, \\ Charles University Medical School Teaching Hospital, Hradec Králové, Czech Republic
}

\begin{abstract}
Increased urinary neopterin has been associated with advanced disease and poor prognosis in patients with colorectal carcinoma, but less is known about urinary neopterin in other gastrointestinal tumors. We have investigated urinary neopterin in 53 patients with carcinomas of the upper gastrointestinal tract, pancreas and biliary tract. Compared to controls, urinary neopterin was increased in patients with gastric carcinoma, pancreatic carcinoma, cholangiocarcinoma and gallbladder carcinoma. Urinary neopterin was a significant prognostic indicator of survival in univariate as well as multivariate analyses. No significant change in urinary neopterin concentrations was observed during the short follow-up, but increased urinary neopterin concentration at the end of followup was associated with poor prognosis. In conclusion, significantly increased urinary neopterin concentrations were observed in the present cohort of patients. Urinary neopterin is an independent prognostic indicator in patients with carcinomas of the upper gastrointestinal tract, biliary tree and pancreas.
\end{abstract}

Key words: gallbladder carcinoma, gastric carcinoma, cholangiocarcinoma, neopterin, pancreatic carcinoma

\section{Introduction}

Malignant tumors are known to elicit host response mediated by both adaptive and innate immune systcms. Among other laboratory parameters, advanced tumors are associated with increased concentrations of neopterin in the urine or in serum $(1,2)$. Neopterin is produced by macrophages activated by interferon- $\gamma$ (IFN- $\gamma$ ), and elevated neopterin concentrations have been observed in paticnts with different primary tumors, including gastrointestinal carcinomas. Urinary or serum neopterin concentrations have been studied in patients with colorectal carcinoma (3-5), and increased urinary neopterin has been associated with advanced disease (3) or poor prognosis (5). Much less is known about neopterin concentrations in patients with gastrointestinal tumors other than colorectal carcinoma (6).

Gastric carcinoma, pancreatic carcinoma and biliary tract carcinomas (gallbladder carcinoma and cholangiocarcinoma) occur in Europe with significantly lower frequency compared with colorectal carcinoma
(7). Compared to colorectal carcinoma, the prognosis of these tumors is poor, and most patients die of the disease within one or two years of diagnosis. In the present study, we have evaluated the urinary ncopterin in patients with esophageal, gastric, pancreatic and biliary carcinomas.

\section{Material and Methods}

A retrospective analysis was performed of urinary neopterin measurements collected between 1997 and 2000 as a part of research project, approved by the institutional ethics committee, evaluating immune system activation in patients with liver tumors. Morning urine samples were collected from 53 patients, 30 males and 23 females, aged $57 \pm 13$ (range 26 - 79) years. with histologically verified carcinomas of the esophagus, stomach, pancreas, gallbladder or bile ducts, treated at the Department of Oncology and Radiotherapy, Charles University Medical School Teaching Hospital, and 33 healthy controls, 16 males

Correspondence to: Bohuslav Melichar M.D., Ph.D., Klinika onkologie a radioterapie, Lékarská fakulta Univerzity Karlovy a Fakuitni nemocnice, Budova 23, Sokolská 581, 50005 Hradec Králové, Czech Republic phone: +420-49-5834574; fax:+420-49-5832081; e-mail: melichar@fnhk.cz

Pteridines/Vol. 17/No. 1 
Table 1. Urinary neopterin concentrations ( $\mu \mathrm{mol} / \mathrm{mol}$ creatinine) in patients with cancer and in healthy controls

\begin{tabular}{|l|c|c|c|c|c|c|}
\hline \multicolumn{1}{|c|}{ Primary } & $\mathrm{n}$ & Mean \pm S.D. & Range & $\begin{array}{c}\text { Patients with } \\
\text { neopterin } \geq 170 \\
\mathrm{n}(\%)\end{array}$ & $\begin{array}{c}\text { Patients with } \\
\text { neopterin } \geq 214 \\
\mathrm{n}(\%)\end{array}$ & $\begin{array}{c}\mathrm{P} \\
\text { (vs. controls) }\end{array}$ \\
\hline Gallbladder carcinoma & 9 & $443 \pm 626$ & $83-2080$ & $5(56)$ & $5(56)$ & 0.002 \\
\hline Cholangiocarcinoma & 16 & $168 \pm 83$ & $70-361$ & $5(31)$ & $4(25)$ & 0.004 \\
\hline Pancreatic carcinoma & 13 & $204 \pm 116$ & $42-481$ & $8(62)$ & $6(46)$ & 0.003 \\
\hline Gastric carcinoma & 14 & $201 \pm 181$ & $47-767$ & $6(43)$ & $4(29)$ & 0.02 \\
\hline Esophageal carcinoma & 1 & 721 & - & $1(100)$ & $1(100)$ & - \\
\hline All patients & 53 & $243 \pm 296$ & $42-2080$ & $25(47)$ & $20(38)$ & 0.00003 \\
\hline Controls & 33 & $110 \pm 52$ & $34-293$ & $3(9)$ & $2(6)$ & - \\
\hline
\end{tabular}

and 17 females, aged $49 \pm 13$ (range $28-88$ ) years. Thirty-four patients were chemotherapy-naive, 19 patients had previously received therapy. Twenty-nine patients had metastatic disease (including liver metastases) at the time of investigation.

Urinary neopterin was measured by high-performance liquid chromatography. Creatinine was determined by Jaffé reaction, and the results were expressed as neopterin/creatinine ratio ( $\mu \mathrm{mol} / \mathrm{mol}$ crcatininc) as described earlier (8). Peripheral blood cell count was performed as described (9). Hemoglobin was measured by a photometric method using sodium lauryl sulfate, leukocytes were detected by flow cytometry and platelets by impedance method using a Sysmex $\mathrm{XE}$ 2100 blood analyzer (Sysmex, Kobe, Japan).

The survival of patients was analyzed by the Kaplan-Meier method, and the comparisons of survival of patients with urinary neopterin $<$ or $\geq 170$ $\mu \mathrm{mol} / \mathrm{mol}$ creatinine and other survival comparisons were performed by log-rank test. The cutoff value of $170 \mu \mathrm{mol} / \mathrm{mol}$ creatinine was selected based on our previous study (10). The survival was measured from the date of neopterin determination. Urinary neopterin concentrations of patients and controls were compared by Mann-Whitney $U$ test, neopterin at the start and at the end of follow-up was compared by Wilcoxon signed rank test, and correlations between urinary ncopterin and hematological parameters were investigated by Spearman's rank correlation. Multivariate analysis was performed using the Cox regression analysis with the results expressed as hazard ratio (HR). The decision on statistical significance was based on $p<0.05$ significance level. The analyses were performed using NCSS 2001 software (Number Cruncher Statistical Systems, Kaysville, Utah, USA).

Table 2. Univariate analysis of prognostic indicators

\begin{tabular}{|c|c|c|c|}
\hline Parameter & $\begin{array}{c}\text { Cutoff for survival } \\
\text { analysis }\end{array}$ & $\begin{array}{l}\text { Median survival } \\
\text { (months) }\end{array}$ & P (log-rank test) \\
\hline $\begin{array}{l}\text { Urinary neopterin } \\
\text { ( } \mu \mathrm{mol} / \mathrm{mol} \text { creatinine) }\end{array}$ & $\begin{array}{c}<170(\mathrm{n}=28) \text { vs. } \\
\geq 170(\mathrm{n}=25)\end{array}$ & 12 vs. 5 & 0.008 \\
\hline Leukocytes $\left(10^{9} / 1\right)$ & $\begin{array}{c}<8(\mathrm{n}=21) \text { vs. } \\
\geq 8(\mathrm{n}=32)\end{array}$ & 11 vs. 6 & 0.06 \\
\hline Hemoglobin $(\mathrm{g} / 1)$ & $\begin{array}{c}<125(\mathrm{n}=33) \text { vs. } \\
\geq 125(\mathrm{n}=20)\end{array}$ & 6 vs. 13 & 0.03 \\
\hline $\begin{array}{l}\text { Mean erythrocyte } \\
\text { volume (fl) }\end{array}$ & $\begin{array}{l}<84(\mathrm{n}=13) \text { vs. } \\
\geq 84(\mathrm{n}-40)\end{array}$ & 4 vs. 8 & 0.10 \\
\hline Platelets $\left(10^{9} / 1\right)$ & $\begin{array}{c}<270(\mathrm{n}=23) \text { vs. } \\
\geq 270(\mathrm{n}=30)\end{array}$ & 10 vs. 7 & 0.13 \\
\hline Previous therapy & $\begin{array}{c}\text { yes }(=19) \text { vs. } \\
\text { no }(n=34)\end{array}$ & 7 vs. 7 & 0.68 \\
\hline Distant metastases & $\begin{array}{c}\text { no }(n=24) \text { vs. } \\
\text { yes }(n=29)\end{array}$ & 11 vs. 6 & 0.05 \\
\hline Primary & $\begin{array}{c}\text { non-biliary }(\mathrm{n}=28) \\
\text { vs. biliary }(\mathrm{n}=25)\end{array}$ & 7 vs. 11 & 0.03 \\
\hline
\end{tabular}




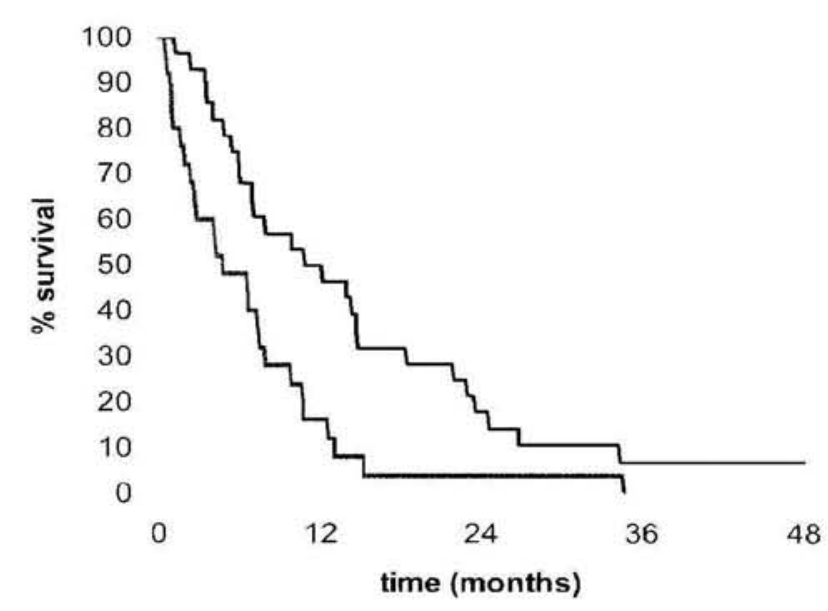

Figure 1. Survival of patients with urinary neopterin $<170 \mu \mathrm{mol} / \mathrm{mol}$ creatinine (bold line) and $\geq 170$ $\mu \mathrm{mol} / \mathrm{mol}$ creatinine (dashed line)

\section{Results}

Urinary neopterin was significantly increased compared to controls in the whole patient group as well as in patients with gallbladder carcinoma, cholangiocarcinoma, pancrcatic carcinoma or gastric carcinoma (Tab. 1). A significant percentage of patients had urinary neopterin equal or above $170 \mu \mathrm{mol} / \mathrm{mol}$ creatinine and equal or above $214 \mu \mathrm{mol} / \mathrm{mol}$ creatinine (mean +2 standard deviations of controls). No statistically significant difference was observed in urinary neopterin levels among patients with different primary tumors, in patients with or without metastases (192 \pm 145 $\mu \mathrm{mol} / \mathrm{mol}$ creatinine vs. $305 \pm 406 \mu \mathrm{mol} / \mathrm{mol}$ creatinine) as well as in chemotherapy-naive patients and patients previously treated with chemotherapy (194 \pm $118 \mu \mathrm{mol} / \mathrm{mol}$ creatinine vs. $330 \pm 463 \mu \mathrm{mol} / \mathrm{mol}$ creatinine). A significant correlation was observed between urinary neopterin and the age of the patients $\left(r_{s}=0.44\right.$. $\mathrm{p}=0.001)$ and peripheral blood leukocyte count $\left(\mathrm{r}_{\mathrm{s}}=\right.$ $0.27, p=0.05$ ), but not with hemoglobin, mean erythrocyte volume and peripheral blood platelet count.

Median survival of all paticnts was 7 months ( 7 months in patients with gastric carcinoma, 8 months in pancreatic carcinoma and 11 months in patients with biliary tract carcinoma). In univariate analysis, urinary neopterin below $170 \mu \mathrm{mol} / \mathrm{mol}$ creatinine, hemoglobin concentration, biliary primary and presence of distant metastases (including liver metastases) were significant indicators of survival, and peripheral blood lcukocyte count was of borderline significance (Table 2). In multivariate analysis, only urinary neopterin below $170 \mu \mathrm{mol} / \mathrm{mol}$ creatinine (HR 0.39, $\mathrm{p}=0.002$ ) and absence of distant metastases (HR 0.49, p =0.02) werc significant indicators of survival.
In 26 patients, urinary neopterin was determined on more than one occasion. The mean $( \pm$ standard deviation) number of neopterin measurements was $5 \pm 3$ (range 2 - 16), and mean time between the earliest and latest measurement was $3.5 \pm 3.2$ (range 0.1 - 13) months. No significant change in urinary neopterin was observed between the earliest (234 \pm 285 $\mu \mathrm{mol} / \mathrm{mol}$ creatinine $)$ and latest $(214 \pm 154 \mu \mathrm{mol} / \mathrm{mol}$ creatinine) measurements. In patients with urinary neopterin at the time of the latest measurement equal or above $170 \mu \mathrm{mol} / \mathrm{mol}$ creatinine significantly shorter survival was observed (median 4 vs. 12 months, $\mathrm{p}=$ $0.05)$, but in some patients specimens were collected during therapy, in others rather shortly before death.

\section{Discussion}

In the present study significantly increased urinary neopterin concentrations were observed, compared to controls, in the whole group of patients as well as in patients with gallbladder carcinoma, cholangiocarcinoma, pancreatic carcinoma, or gastric carcinoma. This observation was not unexpected as elevated urinary or serum neopterin concentrations have been reported in patients with wide range of tumors (2). Similarly to other cancers, increased neopterin in the present study was significant indicator of poor prognosis on univariate analysis both at the start and at the end of follow-up, as well as on the multivariate analysis. Both chemotherapy-naive patients as well as patients undergoing chemotherapy were included in the present study (with the sample collected before the next chemotherapy cycle). In another study (Melichar et al. manuscript in preparation), we have demonstrated that the chemotherapy regimens used in gastrointestinal cancer (usually based on 5-fluorouracil) do not lead to a significant change in urinary neopterin, we therefore included in the present analysis also the patients who have already been treated with chemotherapy.

Neopterin is an unconjugated pteridine synthesized from guanosine triphosphate (GTP). GTP is converted to 7.8-dihydroneopterin by the enzyme GTP cyclohydrolase I, and this reaction represents the first step in a pathway leading to the formation of tetrahydrobiopterin (1). GTP cyclohydrolase I is the rate-limiting enzyme in this pathway, and its activity is induced by IFN- $\gamma$ (11), that is produced by T-lymphocytes and natural killer (NK) cells (12). Human macrophages possess significant GTP cyclohydrolase I activity when stimulated by IFN- $\gamma$, while the activities of other enzymes of this pathway are low (13), leading to the accumulation of 7,8-dihydroneopterin-triphosphate which undergoes autooxidation to neopterin.

Among gastrointestinal tumors, neopterin concen- 
trations have been studied most extensively in patients with colorectal carcinoma. In patients with colorectal carcinoma neopterin concentrations were associated with advanced stage (3), poor prognosis (5), and correlated with the decrease of serum tryptophan and decreased quality of life (14). Increased serum or urinary neopterin concentrations have also been reported in patients with hepatocellular carcinoma $(15,16)$. Kawasaki et al. (16) observed increased urinary neopterin in 18 patients with hepatocellular carcinoma. A significant correlation was observed between urinary neopterin and tumor size, and survival was significantly shorter in patients with higher urinary neopterin.

Much less is known about the presence and prognostic impact of increased neopterin concentrations in patients with other gastrointestinal tumors. In pilot studies, high urinary neopterin concentrations have been observed in substantial number of patients with gastric carcinoma and most patients with pancreatic carcinoma (6). Manes et al. (17) reported markedly increased serum neopterin in patients with pancreatic carcinoma. Increased serum neopterin concentrations were also reported in patients with pancreatic carcinoma by Birk et al. (18). Contrary to other reports in patients with malignant tumors as well as to the present report, in the study reported by Birk et al. (18) higher serum neopterin has been associated with better prognosis. In another study in patients with gastric and colorectal carcinomas, the urinary neopterin concentrations in patients with gastric carcinoma were similar to paticnts with colorectal carcinoma (19). Even less is known about neopterin in patients with biliary tract carcinoma (gallbladder carcinoma and cholangiocarcinoma). In onc report published so far, increased urinary neoptcrin has becn observed in 8 out of 9 patients examined (6), and present data are in agreement with this report, although the number of patients with increased urinary ncoptcrin was lower in the present series.

Limited number of patients with each primary was evaluated in the present study, and the data were pooled for survival analysis for all patients. In clinical studies, patients with gallbladder carcinoma and cholangiocarcinoma are almost invariably handled together as a single group and, because of a relatively low frequency of these tumors, biliary tract carcinomas are often pooled together with other gastrointestinal carcinomas. Because of the relatively low incidence, carcinomas of the biliary tract and pancreas have been studied together even in prospective clinical trials (20). This approach may be justified as prognosis and chemosensitivity of these tumors is similar. Morcover, in some cases it may be difficult to distinguish cholangiocarcinoma from pancreatic carcinoma, and the diagnosis of cholangiocarcinoma relies on the exclu- sion of a primary tumor elsewhere in the gastrointestinal tract (21), notably in the pancreas or stomach. Inclusion of patients with upper gastrointestinal of pancreaticobiliary carcinomas in one study may be further justified by the fact that the site of the primary may be determined only after extensive studies, and an information about prognosis could be helpful at the start of patient evaluation. However, future studies on a larger cohort of patients should address the prognostic significance of increased urinary neopterin in patients with individual primary tumors in this group.

Advanced cancer is often accompanied by anemia that may be further aggravated by antitumor therapy. In the majority of cancer patients, the ancmia may be categorized as anemia of chronic disease, characterized by low serum iron, low serum iron binding capacity and high ferritin (22). The decline of hemoglobin concentrations in anemia of chronic disease results from the alterations of iron metabolism associated with acute phase response and direct inhibition of hematopoietic progenitors by cytokines responsible for systemic inflammatory and immune response, e.g. IFN- $\gamma$ or tumor necrosis factor- $\alpha$ (22), and decreased hemoglobin levels are associated with increased neopterin concentrations. A negative correlation between serum neopterin concentrations and hemoglobin has been observed in patients with hematological and gynecological malignancies $(23,24)$. In contrast to observations made in patients with other primary tumors, no correlations were observed between hemoglobin or mean erythrocyte volume, an indicator of ancmia of chronic disease, and urinary neopterin. This lack of correlation may be explained by the possibility that anemia in these patients may be caused or aggravated by factors other than systemic inflammatory or immune response, e.g. chronic gastrointestinal blood loss or, in previously treated patients, by chemotherapy. The correlation between urinary neopterin and peripheral blood leukocyte counts reflects an association between different parameters of systemic inflammatory and immune response. The significant correlation observed between urinary neopterin and the age of the patients is in agreement with our earlier study demonstrating increased concentrations and prognostic significance of urinary neopterin in the elderly (9).

In conclusion, increased urinary neopterin was observed in patients with gastric carcinoma, pancreatic carcinoma, cholangiocarcinoma and gallbladder carcinoma. Urinary neopterin is an independent prognostic indicator in patients with carcinomas of the upper gastrointestinal tract, biliary tree and pancreas. 


\section{Acknowledgement}

Supported by Research Project MZO 00179906.

\section{References}

1 Wachter H, Fuchs D, Hausen A, Reibnegger G. Werner ER. Neopterin as marker for activation of cellular immunity: immunologic basis and clinical application. Adv Clin Chem 1989; $27: 81-141$.

2 Reibnegger G, Fuchs D, Fuith LC, et al. Neopterin as a marker for activated cell-mediated immunity: application in malignant disease. Cancer Detect Prev 1991; 15: 483 - 490.

3 Melichar B, Jandik P, Solichova D, et al. Urinary neopterin excretion in colorectal cancer. Onkologie $1994 ; 17: 434$ - 436.

4 Allen-Mersh TG, Glover C, Fordy C, Henderson DC, Davies M. Relation between depression and circulating immune products in patients with advanced colorectal cancer. J Roy Soc Med 1998; 9: $408-413$.

5 Weiss G, Kronberger P, Conrad F, Bodner E, Wachter H, Reibnegger G. Neopterin and prognosis in patients with adenocarcinoma of the colon. Cancer Res 1993; 53: 260 - 265.

6 Conrad F, Bodner E, Fuchs D, et al. Determination of neopterin - a marker of cellular immunity - in gastrointestinal and pancreatic carcinoma. In: Pfleiderer W, Wachter H, Curtius C, eds. Biochemical and Clinical Aspects of Pteridines. Berlin-New York: Walter de Gruyter 1984: 357 366.

7 Levi F, Lucchini F, Negri E, Boyle P. La Vecchia C. Cancer mortality in Europe, 1990-1994, and an overview of trends from 1955 to 1994. Eur J Cancer 1999; 35: 1477 - 1516.

8 Cermanová M, Melichar B, Solichová D, Bláha M, Bláha V, Blazek M, Mašín V, Cerman J, Zadák Z. Urinary neopterin and microalbuminuria in patients treated by low-density lipoprotein apheresis. Pteridines 2005; 16: 174 - 183.

9 Solichova D, Melichar B, Blaha V, et al. Biochemical profile and survival in nonagenarians. Clin Biochem 2001; 34: 563 - 569.

10 Melichar B, Solichova D, Svobodova I, Melicharova K. Neopterin in renal cell carcinoma: inhalational administration of interleukin-2 is not accompanied by a rise of urinary neopterin. Luminescence 2005; 20: 311 - 314.

11 Huber C, Batchelor JR, Fuchs D, et al. Immune response-associated production of neopterin. Release from macrophages primarily under control of interferon- $\gamma$. J Exp Med 1984; 160: 310 - 316.
12 Fuchs D, Hausen A, Reibnegger G, Werner ER, Dierich MP, Wachter H. Neopterin as a marker for activated cell mediated immunity: application in HIV infection. Immunol Today 1988; 9: 150 - 155.

13 Werner ER, Werner-Felmayer G, Fuchs D, et al. Tetrahydrobiopterin biosynthetic activities in human macrophages, fibroblasts, THP-1, and T24 cell. J Biol Chem 1990; 265: 3189 - 3192.

14 Huang $A$, Fuchs D, Widner B, Glover C, Henderson DC, Allen-Mersh TG. Serum trytophan decrease correlates with immune activation and impaired quality of life in colorectal cancer. $\mathrm{Br} \mathrm{J}$ Cancer 2002; 86: 1691 - 1696.

15 Tsuda Y, Fukuda A, Kobayashi H, et al. Serum neopterin as a marker for screening of hepatocellular carcinoma. Pteridines 2004; 15: 161 - 169.

16 Kawasaki H, Watanabe H, Yamada S, Watanabe K, Suyama A. Prognostic significance of urinary neopterin levels in patients with hepatocellular carcinoma. Tohoku J Exp Med 1988; 155:311 - 318.

17 Manes G, Spada OA, Rabitti PG, et al. Neopterin serum levels in pancreatic adenocarcinoma. Int $\mathrm{J}$ Pancreatol 1999; 25: 31 - 37.

18 Birk D, Gansauge F, Gansauge S, Schwarz A, Beger HG. Levels of serum neopterin are increased in pancreatic cancer patients and correlate with the prognosis. Eur J Med Res 1999; 4: 156 - 160.

19 Peters KM, Bornhofen B, Grundmann R, et al. Neopterin als Marker einer unspezifischen Immunstimulation mittels Propionibacterium avidum KP-40 bei Patienten mit gastrointestinalen Tumoren. Med Klin 1990; 85: 421 - 424.

20 Glimelius B, Hoffman K, Sjoden PO, et al. Chemotherapy improves survival and quality of live in advanced pancreatic and biliary cancer. Ann Oncol 1996; 7: $593-600$.

21 Khan SA, Davidson BR, Goldin R, et al. Guidelines for the diagnosis and treatment of cholangiocarcinoma: consensus document. Gut 2002; 51: VI1 - V19.

22 Fuchs D, Hausen A, Reibnegger G, et al. Immune activation and the anaemia associated with chronic inflammatory disorders. Eur J Haematol 1991; 46: $65-70$.

23 Denz H, Fuchs D, Huber $\mathrm{H}$, et al. Correlation between neopterin, interferon-gamma and haemoglobin in patients with haematological disorders. Eur J Haematol 1990; 44: 186 - 189.

24 Wachter H, Artner-Dworzak E, Fuchs D, et al. Association between neopterin and iron metabolism in cancer patients. Tumor Diagn Ther 1990; 11: $229-232$. 